\title{
Construção de tecnologia educativa para pessoas acometidas pela hanseníase
}

\section{Construction of educational technology for people affected by leprosy}

\author{
Rebecca Maria Façanha Sampaio ${ }^{1 *}$, Rones Alves Pinheiro ${ }^{1}$, Danielle Teixeira Queiroz ${ }^{2}$, João
}

Victor Farias Mota ${ }^{3}$, Francisco Gabriel de Andrade Mota ${ }^{3}$, Valéria Freire Gonçalves ${ }^{2}$, Marielene Alves Guanabara², Manoel Domingos Maciel Neto ${ }^{3}$

\begin{abstract}
RESUMO
Objetivo. Construir uma tecnologia educativa (cordel) para orientação sobre Hanseníase. Metodologia. Trata-se de um estudo metodológico realizado entre os anos de 2019 a 2020, composto por seis fases, primeira fase: fundamentação teórica, segunda fase: fichamento e levantamento, terceira fase: montagem dos versos e rimas, quarta fase: diagramação, quinta fase: correção gramatical, sexta fase: impressão. Sendo o cordel apresentado ao fim do trabalho como anexo. Resultados: O cordel é composto por oito estrofes, cada uma delas aborda um fragmento do tema hanseníase. Os assuntos abordados em cada estrofe são historicidade, epidemiologia e importância do tratamento, fisiopatologia, sintomatologia, tratamento, conscientização e combate ao preconceito e inclusão social dos acometidos pela hanseníase. Conclusão. Foi compreendido que a literatura de cordel é viável para educação em saúde e que a tecnologia relatada neste trabalho tem a possibilidade de educar sobre a hanseníase. Já as intuições de saúde e ensino podem incentivar esta tecnologia produzindo maios para produção de mais tecnologias deste porte em suas educações em saúde.
\end{abstract}

Palavras-chave: Hanseníase; Educação em Saúde; Literatura.

\begin{abstract}
Objective. To construct an educational technology (cordel) for orientation about Leprosy. Methodology. This is a methodological study carried out between the years 2019 to 2020, composed of six phases, first phase: theoretical foundation, second phase: fiching and survey, third phase: assembly of verses and rhymes, fourth phase: diagramming, fifth phase: grammatical correction, sixth phase: printing. Being the cordel presented at the end of the work as an appendix. Results. The cordel is composed of eight stanzas, each of which addresses a fragment of the leprosy theme. The subjects addressed in each stanza are history, epidemiology and importance of treatment, pathophysiology, symptoms, treatment, awareness and combating prejudice and social inclusion of people affected by leprosy. Conclusion. It was understood that cordel literature is viable for health education and that the technology reported in this work has the possibility to educate about leprosy. The health and education institutions can encourage this technology, producing more technologies of this size in their health education.
\end{abstract}

Keywords: Leprosy; Health Education; Literature.

\footnotetext{
${ }^{1}$ Enfermeira da Universidade de Fortaleza - UNIFOR - Fortaleza-CE.

*E-mail: rebecca_facanha@ hotmail.com

2 Docente da Universidade de Fortaleza - UNIFOR - Fortaleza-CE.

${ }^{3}$ Bolsista da Universidade de Fortaleza - UNIFOR - Fortaleza-CE.
} 


\section{INTRODUÇÃO}

A hanseníase é uma doença infecto-contagiosa de evolução crônica, causada por um bacilo denominado micobateriumleprae. A doença se manifesta principalmente, por lesões cutâneas com diminuição da sensibilidade térmica, dolorosa e tátil. Tais manifestações se dão pela predileção que o bacilo tem por células cutâneas e terminações nervosas. Pessoas de todas as idades podem ser acometidas, principalmente aquelas na faixa etária economicamente ativa, tendo assim grande importância para a saúde pública, devido à sua magnitude e seu alto poder incapacitante (LYON e GROSSI, 2013).

Em 1873 o médico norueguês Gerhard Armauer Hansen, notável pesquisador sobre o tema, identificou o bacilo como o causador da doença, que até então era chamada de lepra. A partir desta descoberta o micobateriumleprae ficou conhecido como bacilo de HANSEN e a doença passou a se chamar hanseníase, em homenagem ou pesquisador (EIDT, 2004).

De acordo com a OMS, entre 2014 e 2018, foram identificados no território brasileiro 140.578 casos novos de hanseníase. No meio destes, 77.544 casos novos deramse no sexo masculino, o que representa $55,2 \%$ do total. No mesmo intervalo, observou-se predominância desse sexo na maior parte das faixas etárias e anos. O maior número foi constatado na população entre 50 a 59 anos, totalizando 26.245 casos novos. Nesse tempo, percebeu -se que a taxa de detecção por 100 mil habitantes na população masculina foi superior que na população feminina em todas as faixas etárias, especialmente a partir dos 20 anos de idade, excetuando as faixas de 10 a 14 anos. Esse índice é crescente com o aumento da faixa etária, apresentando, nos indivíduos masculinos de 70 a 79 anos de idade, uma taxa média de detecção cerca de cinco vezes maior que na população de 10 a 14 anos (OMS, 2020).

Para que haja uma maior sensibilização da sociedade quanto à causa da hanseníase, é necessário que o conhecimento sobre esta doença seja difundido, neste sentido as tecnologias auxiliam os profissionais da saúde na realização deste trabalho. Sendo assim, o cordel com o tema hanseníase é capaz de auxiliar neste trabalho, visto sua fácil adesão, compreensão, aplicação e baixo custo (SESA, 2020). 
As tecnologias leves são conhecidas como características da relação humana do cuidado, identificada no âmbito da enfermagem como o conjunto de relações que representam o cuidar em si, cujo contato entre o profissional e o paciente acontece de forma direta, com conexão interpessoal, ou seja, troca de aprendizado entre os envolvidos. Assim, o atendimento e a relação/interação são termos definidores dessa tecnologia (SABINO et al., 2016).

$\mathrm{Na}$ literatura de cordel, os contos passaram a ser apresentados em versos, impecavelmente metrificados, que deviam ser narrados quase que em forma cantada, em harmonia que satisfaz leitores e ouvintes em todo o mundo. Para Galvão (2006) os folhetos de Cordéis são expressões literárias principalmente nordestinas e que emprega um tipo de poesia equivalente à poesia oral tradicional de algumas regiões do Nordeste. Contudo, a autora destaca que existe uma desigualdade clara entre essas duas tradições, a oral e a escrita: para que os versos sejam vistos como pertencentes ao gênero da literatura de Cordel, estes devem estar impressos. Os contos encontrados nos folhetos retratam os padrões da cultura onde foi criada, apresentando como é a vida desse povo, suas sociedades e suas religiões. Mais do que simples editoriais destinados a pessoas simples, os folhetos de Cordéis tornaram-se ícones específicos da cultura nordestina, estudados por pesquisadores de diferentes áreas do conhecimento (SILVA, 2012).

O objetivo deste trabalho foi construir uma tecnologia educativa (cordel) para orientação de pessoas acometidas pela hanseníase.

\section{METODOLOGIA}

Pesquisa do tipo metodológico, que se refere a investigação e aplicação de métodos para obtenção, organização e interpretação dos dados para construção de um cordel, que será avaliado processualmente através de passos implementados e debatidos a cada etapa metodológica. Nesse tipo de investigação, o pesquisador desenvolverá um instrumento educativo (cordel) de fácil aplicabilidade e compreensão, para que possa ser manuseado por outros profissionais que lidam com os com educação em saúde e combate a hanseníase, tal qual é evidenciado na pesquisa de enfermagem (POLIT; BECK; HUNGLER, 2011). 


\section{Fases e período do estudo}

A construção do cordel aconteceu entre os anos de 2019 e 2020 por meio de seis fases, sendo estas, executadas em períodos diferentes, de forma sistematizada e contínua. Cada fase é definida e nomeada conforme sua realização e o objetivo que cumpre no processo de construção. As fases que compõem este são: fundamentação teórica, seleção e fichamento do conteúdo, montagem dos versos e rimas, diagramação, correção gramatical e impressão do cordel.

Para construção de uma tecnologia, no caso o cordel, que tem por objetivo informar e educar, foi necessário a formação de um rico embasamento teórico tendo em vista a

Tendo em vista a complexidade e o vasto conhecimento científico que se tem sobre a hanseníase. Foi realizada uma busca em bases de dados nacionais e delas extraídas alguns artigos científicos, periódicos e manuais que tratam sobre a doença. Assuntos como epidemiologia, fisiopatologia, transmissibilidade, sintomatologia e tratamento foram destaques no material colhido e a esta fase, dá-se o nome de embasamento teórico.

Após esta etapa, iniciou-se a segunda fase, que foi o processamento destas informações aonde ocorreu a seleção e o fichamento dos principais pontos a serem abordados nos versos do cordel. Para o devido êxito desta etapa foi necessário identificar os assuntos relevantes ao conhecimento básico sobre a hanseníase, dentre eles foram elencados os seguintes: epidemiologia, fisiopatologia, sintomatologia, sintomatologia e tratamento, além de questões históricas e sociológicas da doença.

$\mathrm{Na}$ terceira fase aconteceu a montagem dos versos e rimas do cordel, aqui prevalece a capacidade artística do autor, sendo este o principal responsável pela qualidade das rimas e clareza das informações contidas na poesia. Nesta etapa o cordelista (autor do cordel) munido dos assuntos a serem abordados, fez o encaixe de palavras com o intuito de formar frases que tenham uma ligação entre si, esta ligação chama-se rima. Formando um conjunto de quatro ou seis frases, tem-se uma estrofe e o conjunto de mais de uma estrofe forma-se o cordel. 
A diagramação do cordel aconteceu em programa de edição de texto em computador, WORD 2016 Microsoft Office. No primeiro momento sendo o cordel digitado e em segundo momento as frases sendo diagramadas em letra do tipo ARIAL tamanho doze, as frases foram postas em espaçamento um e meio, já todo o cordel foi posto a esquerda da página, com destaque em negrito apenas para o título.

A correção gramatical foi feita por meio de leitura e análise de todo o texto contido no cordel, as palavras e frases foram corrigidas de acordo com a gramática apresentada no dicionário Aurélio. Já a impressão do cordel foi feita em folha de papel branco, de tamanho A4, o layout foi em forma de retrato. O equipamento utilizado foi impressora jato de tinta ligada ao computador por meio de cabo do tipo USB, todo o texto foi impresso em tinta de cor preta.

\section{RESULTADOS E DISCUSSÃO}

O cordel foi composto por oito estrofes, cada uma delas aborda um fragmento do tema hanseníase. Os assuntos abordados em cada estrofe são historicidade, epidemiologia e importância do tratamento, fisiopatologia, sintomatologia, tratamento, conscientização e combate ao preconceito e inclusão social dos acometidos pela hanseníase

De uma forma bem sucinta a primeira estrofe do cordel aborda três assuntos sobre a hanseníase, o tempo de existência desta afecção, a sua existência nos dias de hoje e a importância de tratar esta enfermidade. Ao falar do tempo de existência da doença, o cordel entra em concordância com o que diz o autor (OPROMOLLA, 2000), onde ele fala que os primeiros relatos de hanseníase datam do ano de 1500 a. c. em um livro sagrado na Índia, chamado Regveda Samhita.

Já ao falar sobre a existência da doença nos dias de hoje o cordel entra em consenso com o que diz o Ministério da saúde (MS) no ano de 2021, que entre os anos de 2015 a 2019 houve o diagnóstico de 137.385 novos casos de hanseníase no Brasil. Sobre a importância de tratar esta doença o cordel reforça o que diz a OMS (Organização Mundial de Saúde) em sua estratégia global para hanseníase 2016-2020, onde no segundo pilar fala sobre o reforço nas medidas de adesão ao tratamento e consequentemente a redução de incapacidades provocadas por esta infecção (OMS, 2016; DATASUS, 2021). 
A estrofe subsequente aborda a fisiopatologia da hanseníase destacando o agente etiológico e o que este causa ao organismo, desde danos primários até acometimentos tardios, no caso as incapacidades. Joel Lastória nos mostra que a hanseníase é uma doença causada por um bacilo chamado micobateriumleprae (M. lepraee), este por sua vez infecta o homem e tem predileção pelas células do sistema nervoso localizado nas periferias do corpo, visto que essas são mais frias. $\mathrm{O}$ acometimento de nervos periféricos trará ao indivíduo alterações cutâneas, alteração de sensibilidade, deformidades entre outras e se não tratados em tempo hábil poderá acarretar em incapacidade física (LASTÓRIA, 2012).

O terceiro parágrafo do cordel fará uma abordagem sobre a transmissibilidade e o poder de infecção do bacilo no organismo. Aqui discutiremos o modo de transmissão da hanseníase que segundo o ministério da saúde se dá por meio das vias aéreas. O indivíduo contaminado expeli por meio da fala, respiração ou tosse o patógeno no ar, pessoas saudáveis que terão contato por longo período com esse ar, irão se contaminar e desenvolverão ou não os sintomas de hanseníase, tal fato ocorre porque algumas pessoas possuem resistência ao M. Leprae. Esse mecanismo de defesa presente no organismo nos será mostrado pelo desenvolvimento da sintomatologia, onde indivíduos com nenhuma resistência desenvolverão a forma grave da doença ao qual denominasse multibacilar, indivíduos com resistência moderada desenvolverão a forma branda cujo nome é paucibacilar e pessoas resistentes ao patógeno em estudo não desenvolveram nenhum sintoma. Este último é o que mais preocupa as autoridades sanitárias, pois por não saber que está contaminado, mantem a cadeia de transmissão ativa (BRASIL, 2017).

O cordel seque a quarta estrofe abordando as características das alterações cutâneas desenvolvidas pela hanseníase, que popularmente são conhecidas como manchas. Em seu livro as autoras Lyon e Grossi nos mostram que as manchas apresentadas na hanseníase em sua maior parte são hipocrômicas ou marrons, podem surgir também pápulas, infiltrações, tubérculos e nódulos. Nestas lesões não há crescimento de pelo e nem presença de suor, além da perda de sensibilidade. Todas estas características são devidas ao acometimento causado pelo bacilo (LYON e GROSSI, 2013).

A quinta estrofe aborda parte do diagnóstico, que é feito em um primeiro momento no consultório por meio de anamnese e exame físico e em um segundo momento em 
laboratório por meio da baciloscopia. Segundo Alba Lúcia a anamnese consiste em um bloco de perguntas a serem realizadas, com o objetivo levantar informações suficientes para conhecer a vida e o histórico do paciente, dentre as indagações estão perguntas de cunho socioeconômico, histórico familiar, história de saúde entre outras. (BARROS, 2016)

A sexta estrofe fala sobre o teste de sensibilidade, sendo este, feito no consultório de forma manual com ajuda de alguns instrumentos, sendo este, o mais acessível para o diagnóstico da hanseníase. O autor Cyro Festa Neto nos mostra em seu livro que o teste é realizado com o paciente de olhos fechados e o profissional tocando suas manchas com o objetivo identificar alguma perda de sensibilidade, sendo assim testada a sensibilidade térmica com dois tubos de ensaio um com água morna e outro com água fria, a sensibilidade dolorosa com um objeto ponte agudo a sensibilidade tátil passando um chumaço de algodão nas lesões (NETO, 2013).

A sétima estrofe do cordel trará uma abordagem do tratamento da hanseníase, falando que o tratamento que é medicamentoso e de sua longa duração, precisando assim de uma adesão por parte do paciente. Todas estas informações são embasadas no que diz o Ministério da saúde em suas diretrizes para vigilância, atenção e eliminação da hanseníase, onde lá consta dois tipos de esquema utilizados. O primeiro para os casos na forma paucibacilar em adultos, que serão utilizados os medicamentos Rifampicina (RFM): dose mensal - 600 mg (2 cápsulas de $300 \mathrm{mg}$ ) com administração monitorada. Dapsona (DDS): dose mensal de $100 \mathrm{mg}$ monitorada e dose diária de $100 \mathrm{mg}$ autoadministrada e nos casos multibacilares será utilizado Rifampicina (RFM): dose mensal de $600 \mathrm{mg}$ (2 cápsulas de $300 \mathrm{mg}$ ) com administração monitorada. Dapsona (DDS): dose mensal de $100 \mathrm{mg}$ monitorada e dose diária de $100 \mathrm{mg}$ auto administrada. Clofazimina (CFZ): dose mensal de $300 \mathrm{mg}$ (3 cápsulas de $100 \mathrm{mg}$ ) com administração monitorada e 1 dose diária de $50 \mathrm{mg}$ auto administrada. Na primeira forma o tratamento durará de seis a nove meses, já na segunda forma o tratamento durará de doze a dezoito meses (BRASIL, 2016).

A oitava estrofe do cordel nos mostra uma reflexão sobre a inclusão dos portadores de hanseníase que iniciaram o tratamento na sociedade, visto que depois de vinte e quatro horas do início do tratamento o indivíduo não transmite mais. Em concordância com esta reflexão, Finotti nos mostra a importância da inclusão social, pois o indivíduo quando 
recebe o diagnóstico de hanseníase tende a se sentir triste e excluído. Tal fato gera uma alta incidência e aparecimento de sintomas depressivos nesta população, 43,1\% em comparação com a população em geral. Visto isso são necessárias políticas de inclusão e até mesmo o próprio convívio deste paciente o chamar para uma inclusão efetiva, sem preconceitos ou estigmas (FINOTTI, 2020).

\section{CONCLUSÃO}

Este estudo permitiu construir a tecnologia proposta e a partir dela compreender que a literatura de cordel tem probabilidade e pode fazer parte dos meios para realização de educação em saúde, visto sua capacidade de atrair atenção e repassar informações complexas de forma lúdica e agradável ao público ao qual se objetiva educar. Esta ferramenta educativa permite falar de vários temas da saúde, abordando vários aspectos da temática a ser trabalhada, vale salientar ainda o seu baixo custo de produção e sua fácil disseminação.

A tecnologia produzida e relatada neste trabalho, aborda a hanseníase de uma forma simples, porém rica em informações, pois fala de vários aspectos da doença, dentre eles podemos citar, historicidade, transmissão, fisiopatologia, sintomatologia, agravos e aspectos sociais. Além de em todo seu contexto incentivar o leitor a refletir sobre sua saúde e a ser um propagador da prevenção e tratamento contra a hanseníase.

Ferramentas como esta permitem os profissionais de saúde abordar assuntos complexos de forma simples e resumida, fazendo assim que o público possa absorver melhor e consequentemente aderir de forma adequada a terapêutica proposta. Tal ferramenta é acessível a qualquer serviço de saúde visto seu baixo custo de produção, além de ser bem aceito na região norte e nordeste por já fazer parte da cultura desta região.

Por fim visto o potencial desta ferramenta é de extrema importância que as instituições de ensino e de saúde promovam a literatura de cordel em suas práticas. Podendo incentivar oficinas, eventos, incentivos financeiros e estruturais e até mesmo treinamento para aperfeiçoar e descobrir possíveis talentos entre os membros de sua equipe. 


\section{REFERÊNCIAS}

BARROS, A. L. B. L. de (org.); Anamnese e exame físico: avaliação diagnóstica de enfermagem no adulto. 3. ed. Porto Alegre: Artmed, 2016. E-book:

https://integrada.minhabiblioteca.com.br/\#/books/9788582712924/cfi/1!/4/4@0.00:54.5. Acesso em: 03 abr. 2021.

EIDT, L. M. Breve história da hanseníase: sua expansão do mundo para as Américas, o Brasil e o Rio Grande do Sul e sua trajetória na saúde pública brasileira. Saúde e Sociedade, São Paulo, vol. 13, n. 2, maio/ago. 2004. Disponível em: https://www.scielo.br/scielo.php?script=sci_arttext\&pid=S0104$12902004000200008 \& \operatorname{lng}=$ pt\&tlng=pt. Acesso em: 03 abr. 2021.

FINOTTI, R. de F. C.; ANDRADE, A. C. de S.; SOUZA, D. P. O. de; Transtornos Mentais Comuns e Fatores Associados Entre Pessoas Com Hanseníase: análise transversal em Cuiabá. Epidemiologia e Serviços de Saúde. Brasília, vol. 29, n. 04, jul. 2020. Disponível em: https://www.scielo.br/scielo.php?script=sci_arttext\&pid=S223796222020000400300\&lng=pt\&nrm=iso\&tlng=pt. Acesso em: 03 abr. 2021.

LASTÓRIAI, J. C; ABREU, M. A. M. M.; Hanseníase: diagnóstico e tratamento. Revista Diagnóstico e Tratamento. São Paulo, vol. 17, ed. 04, p. 173 - 179, out/nov/dez 2012.

LAYON, S.; GROSSI, M. A. de F.; HANSENÍASE. Rio de Janeiro: MedBook, 2013. E-book. Disponível em:

https://integrada.minhabiblioteca.com.br/\#/books/9786557830321/cfi/4!/4/2@100:0.00. Acesso em: 03 abr. 2021.

MS. Boletim Epidemiológico Hanseníase. Brasília: Ministério da Saúde, 2021.

MS. Diretrizes para vigilância, atenção e eliminação da hanseníase como problema de saúde pública. Brasília: Ministério da Saúde, 2016. E-book. Disponível em: https://portalarquivos2.saude.gov.br/images/pdf/2016/fevereiro/04/diretrizeseliminacao-hanseniase-4fev16-web.pdf. Acesso em: 03 abr. 2021.

MS. GUIA PRÁTICO SOBRE A HANSENÍASE. Brasília: Ministério da Saúde, 2017. E-book. Disponível em:

https://portalarquivos2.saude.gov.br/images/pdf/2017/novembro/22/Guia-Pratico-deHanseniase-WEB.pdf. Acesso em: 03 abr. 2021.

NETO, C. F.; CUCÉ, L. C.; REIS, V. M. S. dos; Manual de Dermatologia. 3 ed. Barueri, São Paulo: Manole, 2013. E-book. Disponível em:

https://integrada.minhabiblioteca.com.br/\#/books/9788520451717/cfi/4!/4/4@0.00:57.6. Acesso em: 03 abr. 2021.

OMS. Boletim Epidemiológico Hanseníase. Brasília: Ministério da Saúde, 2020. Ebook. Disponível em: https://antigo.saude.gov.br/images/pdf/2020/May/22/boletimhanseniase-2020-web.pdf. Acesso em: 03 abr. 2021.

OMS; Estratégia Global para Hanseníase 2016-2020: Aceleração rumo a um mundo sem hanseníase. Nova Deli: Organização Mundial da Saúde, 2017. E-book. Disponível 
em: https://apps.who.int/iris/bitstream/handle/10665/254907/9789290225881por.pdf?sequence=8. Acesso em: 03 abr. 2021

OPROMOLLA, D. V. A.; NOÇÕES DE HANSENOLOGIA. Bauru: Centro de Estudos Dr.Reynaldo Quagliato, 2000. E-book. Disponível em:

http://hansen.bvs.ilsl.br/textoc/livros/OPROMOLLA_DILTOR_nocoes/PDF/apres.pdf. Acesso em: 04 abr. 2021.

POLIT, D.F.; BECK, C. T.; Fundamentos de pesquisa em enfermagem: Avaliação de evidências para prática de enfermagem. 9 ed. Porto Alegre: Artmed, 2019. E-book.

Disponível em:

https://integrada.minhabiblioteca.com.br/\#/books/9788582714904/cfi/6/8!/4/2/12/2@0:

0. Acesso em: 04 abr. 2021.

SABINO, L. L. M. de. et al. USO DE TECNOLOGIA LEVE-DURA NAS

PRÁTICAS DE ENFERMAGEM: ANÁLISE DE CONCEITO. Aquichan, Columbia, ano 16, n. 2, jun. 2016. Disponível em:

http://www.scielo.org.co/pdf/aqui/v16n2/v16n2a10.pdf. Acesso em: 04 abr. 2021

SESA. Boletim epidemiológico hanseníse. Secretaria da Saúde do Estado do Ceará, Fortaleza, 2020. Disponível em: https://www.saude.ce.gov.br/wpcontent/uploads/sites/9/2018/06/boletim_hanseniase_10_06_2020_v2.pdf. Acesso em: 04 abr. 2021.

SILVA, A. de O. Clássicos do cordel: desenvolvendo o projeto gráfico de uma coleção de folhetos da literatura de cordel. Orientador: Eduardo Romero Lopes Barbosa. 2012. 87f. Monografia (Trabalho de Conclusão de Curso) - Universidade Federal de Pernambuco, 2012.

\title{
ANEXOS
}

\author{
ANEXO A - CORDEL \\ Hanseníase \\ Das doenças a mais antiga, \\ Porém não está adormecida. \\ A hanseníase pode em mim estar \\ Por isso eu tenho que me olhar \\ E uma ajuda procurar.
}

A hanseníase e causada

Por uma pequena bactéria, 
Podendo até incapacitar

O indivíduo de sua vida continuar.
A bactéria entra em nós,
Por meio da respiração.
$\mathrm{O}$ indivíduo infectado,
Deixará o ar contaminado
Podendo transmitir
Para as pessoas que ali estão,
Que desenvolverão ou não,
Os sintomas dessa infecção

Tudo começa com manchas,

Que podem ser marrons, vermelhas ou brancas.

Ali supor nem pelos vão estar.

E quando eu tocar não vou sentir,

Pois a bactéria já está ali

E os meus nervos acabou de afetar.

As manchas podem estar

Nas pernas, nádegas e cotovelo

Pescoço, costasm braços ou no corpo inteiro.

Pode haver também uma sensação,

De choque ou agulhadas,

Dormência ou formigamento

E em casos mais gravos do nervo um aumento.

No posto de saúde está a solução.

Onde será feitauma investigação, 
Com testes e entrevista,

Teremos pistas e chegaremos a uma conclusão.

Um teste bem simples será realizado,

Onde você ficará de olhos fechados

E o profissional suas manchas irá tocar,

Para ver se você sente ou não.

Daí chegará à conclusão,

Se houve dano nos nervos daquela região.

O importante desta história toda,

É que a hanseníase tem cura.

Todo apoio e ajuda,

Você receberá o tratamento.

São seis meses de medicamento,

Mas você não vai desistir,

Pois no fim da doença

Não estará mais ali.

Outro fato que deve ser comentado

E o preconceito que não deve existir.

Pois depois de 24 horas do início do tratamento,

O indivíduo não transmite mais,

Podendo estar em qualquer local ou ambiente,

No meio da gente,

Recebendo apoio, afeto e muito mais.

E por fim agradeço a sua atenção,

Pedindo a sua colaboração,

Na divulgação desta mensagem 
E se você se identificou com alguma frase,

Peço que haja com coragem

E busque orientação,

Pois nós estaremos de braços abertos

Pra lhe acolher de todo coração.

Recebido em: 15/11/2021

Aprovado em: 10/12/2021

Publicado em: 15/12/2021 